\title{
Calibration and validation of the advanced E-Region Wind Interferometer
}

\author{
S. K. Kristoffersen ${ }^{1}$, W. E. Ward ${ }^{1}$, S. Brown ${ }^{2}$, and J. R. Drummond ${ }^{3}$ \\ ${ }^{1}$ Department of Physics, University of New Brunswick, Fredericton, NB, E3B 5A3, Canada \\ ${ }^{2}$ CRESS Space Instrumentation Laboratory CSIL, York University, 4700 Keele St. Toronto, ON, M3J 1P3, Canada \\ ${ }^{3}$ Department of Physics and Atmospheric Science, Dalhousie University, Halifax, NS, B3H 4R2, Canada
}

Correspondence to: W. E. Ward (wward@unb.ca)

Received: 31 October 2012 - Published in Atmos. Meas. Tech. Discuss.: 14 November 2012

Revised: 8 March 2013 - Accepted: 31 March 2013 - Published: 23 July 2013

\begin{abstract}
The advanced E-Region Wind Interferometer (ERWIN II) combines the imaging capabilities of a CCD detector with the wide field associated with field-widened Michelson interferometry. This instrument is capable of simultaneous multi-directional wind observations for three different airglow emissions (oxygen green line $\left(\mathrm{O}\left({ }^{1} \mathrm{~S}\right)\right.$ ) at a height of $\sim 97 \mathrm{~km}$, the ${ }^{\mathrm{P}} \mathrm{Q}(7)$ and ${ }^{\mathrm{P}} \mathrm{P}(7)$ emission lines in the $\mathrm{O}_{2}(0-1)$ atmospheric band at $\sim 93 \mathrm{~km}$ and $\mathrm{P}_{1}(3)$ emission line in the $(6,2)$ hydroxyl Meinel band at $\sim 87 \mathrm{~km})$ on a three minute cadence. In each direction, for $45 \mathrm{~s}$ measurements for typical airglow volume emission rates, the instrument is capable of line-of-sight wind precisions of $\sim 1 \mathrm{~m} \mathrm{~s}^{-1}$ for hydroxyl and $\mathrm{O}\left({ }^{1} \mathrm{~S}\right)$ and $\sim 4 \mathrm{~m} \mathrm{~s}^{-1}$ for $\mathrm{O}_{2}$. This precision is achieved using a new data analysis algorithm which takes advantage of the imaging capabilities of the CCD detector along with knowledge of the instrument phase variation as a function of pixel location across the detector. This instrument is currently located in Eureka, Nunavut as part of the Polar Environment Atmospheric Research Laboratory (PEARL) $\left(80^{\circ} \mathrm{N}, 86^{\circ} \mathrm{W}\right)$. The details of the physical configuration, the data analysis algorithm, the measurement calibration and validation of the observations from December 2008 and January 2009 are described. Field measurements which demonstrate the capabilities of this instrument are presented. To our knowledge, the wind determinations with this instrument are the most accurate and have the highest observational cadence for airglow wind observations of this region of the atmosphere and match the capabilities of other wind-measuring techniques.
\end{abstract}

\section{Introduction}

Interferometric methods have been used for the past thirty years to passively observe mesospheric and thermospheric winds using Doppler shifts in airglow emissions. Progress in detector technologies have led to advances in the observation capabilities of these instruments by allowing the interference fringes and/or the scene of interest to be imaged. Apart from the Wind Imaging Interferometer (WINDII) on the Upper Atmosphere Research Satellite (Shepherd et al., 1993), publications on working instruments have generally been associated with the Fabry-Perot (Aruhliah et al., 2010; Anderson et al., 2012; Shiokawa et al., 2012) and spatial heterodyne spectroscopy (SHS) techniques (the Doppler Asymmetric Spatial Heterodyne - DASH - of Englert et al., 2012). The success of these instruments demonstrates how incorporating imaging capabilities improves the accuracy and temporal resolution over earlier configurations.

In this paper a configuration which implements these imaging capabilities in combination with a field-widened Michelson interferometer is described. The approach builds on the foundation developed during work on the WINDII instrument. The imaging capability permits simultaneous viewing in multiple directions (as opposed to sequential viewing in each direction) and allows the fringe profile to be imaged and analysed on a bin-by-bin basis using new data analysis algorithms. The resulting instrument, termed the advanced E-Region Wind Interferometer (ERWIN II), generates simultaneous wind observations in 5 directions (the four cardinal directions and zenith) at three different heights using three different emissions (the oxygen green line $(\mathrm{O}(1 \mathrm{~S}))$ 
at $557.7 \mathrm{~nm}$, the ${ }^{\mathrm{P}} \mathrm{P}(7)$ and ${ }^{\mathrm{P}} \mathrm{Q}(7)$ lines in the $\mathrm{O}_{2}(0-1)$ atmospheric band at 865.9 and $866.1 \mathrm{~nm}$ and the $\mathrm{P}_{1}(3)$ emission line in the $\mathrm{OH}(6,2)$ Meinel band at $843.0 \mathrm{~nm})$ every three minutes. The wind accuracy is $\sim 1 \mathrm{~m} \mathrm{~s}^{-1}$ for all the emissions and the precision is $\sim 1 \mathrm{~m} \mathrm{~s}^{-1}$ for the green-line and hydroxyl observations and $\sim 4 \mathrm{~m} \mathrm{~s}^{-1}$ for the $\mathrm{O}_{2}$ observations for standard operating conditions.

ERWIN II is based on the E-region wind interferometer (Gault et al., 1996) which was built in the mid-1990s using a photomultiplier tube as a detector. Winds were obtained by sequentially viewing different directions. This instrument was stationed at Resolute Bay for close to a decade and several papers on the associated observations published (Fisher et al., 2000, 2002; Bhattacharya and Gerrard, 2010).

In 2005, funding through a Canadian Foundation of Innovation grant became available and was used to design and build an improved version of the instrument. The interferometer and filters from the old version of the instrument were retained but the optical configuration and imaging detectors were new. The imaging capability allowed a new data analysis algorithm to be developed, which improved the accuracy and precision of the instrument. Once completed, ERWIN II was moved to the Polar Environment Atmospheric Research Laboratory (PEARL) in Eureka, Nunavut $\left(80^{\circ} \mathrm{N}\right.$, $86^{\circ} \mathrm{W}$ ) in February 2008. It operated satisfactorily for three winters till spring 2011, when some minor issues with its operation occurred. These have been fixed and normal operations resumed in the winter of 2012/2013.

This paper is organized as follows. The next two sections deal with concepts and details necessary for understanding the instrument namely the measurement and instrument concept, and a description of the new instrument and its operation. In the subsequent section, the data analysis algorithms are described. A section on the precision and accuracy of these measurements and their validation follows this. Some initial results along with a comparison with other wind-measuring instruments and a brief review summary of the planned scientific studies with this instrument are then presented. The paper finishes with some concluding remarks.

\section{Measurement and instrument concept}

Doppler shifts in isolated quasi-monochromatic emissions when viewed through an ideal Michelson interferometer are seen as changes in the modulation of the interference fringes as a function of path difference. The measured irradiance $I(\Delta, \lambda, x)$ for an isolated monochromatic emission line has the following form:

$I(\Delta, \lambda, x)=I_{0}\left(1+U V \cos \left(\frac{2 \pi}{\lambda} \Delta+x\right)\right)+I_{\mathrm{B}}$.

Here $I_{0}$ is the irradiance of the emission alone as measured at the detector in the absence of interference, $\Delta$ is the path difference of the interferometer, $\lambda$ is the wavelength of the emission, $x$ represents small variations in path (typically less than $\lambda$ ) that the experimenter can introduce into the interferometer to sample a fringe, $U$ is the relative reduction in fringe amplitude due to instrument effects, $V$ is the relative reduction in fringe amplitude due to the finite width of the emission line and the path difference, and $I_{\mathrm{B}}$ is the sum of the background irradiance of the scene being viewed and detector dark count processes.

The phase, $\theta$, of the fringe is defined as $\frac{2 \pi}{\lambda} \Delta$. A small change in wavelength due to a Doppler shift, $\lambda+\delta \lambda$, results in a change in phase of $\delta \theta=-\left(\frac{2 \pi}{\lambda^{2}}\right) \Delta \delta \lambda$ plus a small correction associated with the dispersion of the glass. For small line-of-sight velocities, $v_{\text {los }}$, the Doppler shift is $\delta \lambda=-\lambda\left(v_{\text {los }} / c\right)$ with positive line-of-sight velocities corresponding to a velocity towards the observer. Hence, the phase change associated with this Doppler shift is

$\delta \theta=\frac{2 \pi \Delta v_{\mathrm{los}}}{c \lambda}$.

Observation of the phase shift relative to the zero-wind phase allows the line-of-sight velocity to be derived.

As described in detail elsewhere (Shepherd, 2002), Doppler Michelson interferometry determines winds by determining phase shifts in the interference by measuring the irradiance passing through the interferometer at specific path differences and determining the fringe parameters from these measurements. Typically, the interferometer path is varied in phase steps of 90 degrees of phase. The standard 4-point algorithm (for ideal conditions: no background or dark count) uses irradiance measurements at four such sequential steps to derive the fringe parameters (irradiance, visibility and phase) as follows:

$$
\begin{aligned}
& I_{0}=\frac{I_{1}+I_{2}+I_{3}+I_{4}}{4} \\
& U V=\frac{\left[\left(I_{1}-I_{3}\right)^{2}+\left(I_{4}-I_{2}\right)^{2}\right]^{1 / 2}}{2 I_{0}} \\
& \theta_{\mathrm{a}}=\tan ^{-1}\left(\frac{I_{4}-I_{2}}{I_{1}-I_{3}}\right) .
\end{aligned}
$$

In passive remote sensing of winds using airglow emissions, the fundamental parameter controlling the measurement quality is the instrument throughput, $A \Omega$, defined as the product of the area, $A$, over which the signal is detected multiplied by the solid angle at the detector, $\Omega$, that the scene of interest subtends. A field-widened Michelson interferometer is a configuration of this interferometer which minimizes the path variation as a function of angle through the interferometer (Shepherd, 2002; details and references pertinent to the following summary can be found in this monograph). Generally the field is collimated through the interferometer so the phase variation as a function of angle through the interferometer is projected onto the observed scene. The field widening is achieved by optimally inserting excess glass into 
one of the arms of the interferometer and positioning the mirror in the other arm at the apparent position of the mirror of the arm with the excess glass in it so that the conditions when the arms are the exactly the same are approximately achieved. This configuration eliminates the second-order dependence of path as a function of angle and can eliminate the fourth-order dependence for specific mirror positions (Zwick and Shepherd, 1971).

The precision of a wind measurement is basically related to how well the sinusoidal variation associated with a variation in path can be discerned relative to the inherent noise associated with that measurement. For a shot-noise-limited observation, a reasonable estimate of accuracy of the phase determination is the standard deviation in $\theta, \sigma_{\theta}$, which is given by Ward (1988):

$\sigma_{\theta}=\frac{1}{a U V \sqrt{I_{0}}}$,

where $a$ is a constant which depends on the number of steps used to determine the fringe phase. For the four point measurement described above $a=\sqrt{ } 2$. For the 8-step scans used for ERWIN II, $a=2$.

The two fringe-related parameters in this equation are the net visibility, $U V$, and $\sqrt{I_{0}}$, which is the signal-to-noise ratio. In practice, the measurement precision can be increased by increasing the signal (longer integration times and enhanced instrument responsivity) or the visibility. The visibility is determined by the emission line width, background emissions, the instrument imperfections and the phase variation over the region used to detect the light (essentially the aperture effect discussed by Hilliard and Shepherd, 1966).

Contributions of background or dark signal to the observed irradiance also affect the measurement precision. In terms of the above formulation for ideal conditions, this can be accommodated by determining effective irradiances and effective visibilities where the effective irradiance is $I_{0}^{\mathrm{e}}=I_{0}+I_{\mathrm{B}}$ and the effective visibility is $U V^{\mathrm{e}}=U V\left(I_{0} /\left(I_{0}+I_{\mathrm{B}}\right)\right)$. In the rest of this paper, for simplicity, expressions will be provided for the ideal conditions (i.e. no dark and no background) with the understanding that for non-ideal conditions, the effective irradiance and visibility can be used without any change to their form.

The wind accuracy is a function of both the measurement precision and knowledge of the phase for a stationary source, termed here the zero-wind phase. Generally, practical sources for the airglow emissions are not available so the zero-wind phase is determined by using lamps with emission lines at wavelengths close to the airglow emissions and using nightly averages of the wind in the vertical direction under the assumption that vertical motions average to a value smaller than the precision.

\section{Instrument description and operation}

ERWIN II differs from the original instrument in that the photomultiplier tube was replaced by an imaging detector, the optics were modified so that light from the four cardinal directions and the vertical could be viewed simultaneously, and the interferometer was placed in an air-tight housing to prevent pressure changes associated with weather fronts from affecting the phase. The interferometer, airglow emissions, calibration lines and the filters used are the same as ERWIN and are described in detail in Gault et al. (1996).

In summary, the interferometer is a three-glass design with the mirror position controlled using piezoelectric cylinders and a capacitive feedback system. The front face is square with sides of $7.62 \mathrm{~cm}$. It was designed to accept a beam with a $2.5^{\circ}$ half angle. The interferometer is set to a path difference slightly larger than $11 \mathrm{~cm}$ and the glass lengths were chosen so that the interferometer is field widened at all wavelengths and thermally compensated. The specific path difference was selected to suppress possible fringes from thermospheric oxygen green-line emissions, and to ensure the fringes of the pair of $\mathrm{O}_{2}$ lines and the $\Lambda$ doubled hydroxyl lines were each in phase. The calibration lines used are the $557.0 \mathrm{~nm}$ krypton line, the $840.8 \mathrm{~nm}$ argon line and the $866.7 \mathrm{~nm}$ argon line.

Figure 1 is a schematic view of the mechanical layout of ERWIN II. It shows a cut through the centre of the instrument such that two of the cardinal viewing directions and the vertical can be seen (the other viewing directions project out of the plane of the figure). The centres of each cardinal viewing direction are at an elevation angle of $38.7^{\circ}$ relative to the horizontal. Light from the sky from all the viewing directions is collected and focused on what is termed the quad mirror, where they are combined into a single beam and then passed through the interferometer. The interferometer is tilted $1.77^{\circ}$ horizontally and $1.77^{\circ}$ vertically relative to the optical axis of the system to ensure that no light was reflected off optical components and recycled back through the system. The optical components which collect the light and collimate it through the interferometer form what is termed the front telescope. After the interferometer, a second optical system termed the back telescope - directs the light to the filters, where the light is again collimated. Both telescopes are $1: 1$. The camera system then focuses the beam onto the detector.

The calibration lamps are located outside of the Michelson housing and are not pictured in the schematic shown in Fig. 1. The lamps are connected to fibre optic cables, which are directed at the calibration lamp screen. This screen acts as a shutter; when it is closed, atmospheric measurements cannot be taken. To take calibration lamp measurements, this shutter/screen is closed, and illuminated by the calibration lamps, and thereby acting as a source for the calibration lines.

The quad mirror consists of four trapezoidal mirrors canted appropriately with a square hole in the middle. This serves to redirect light from each direction so that they 

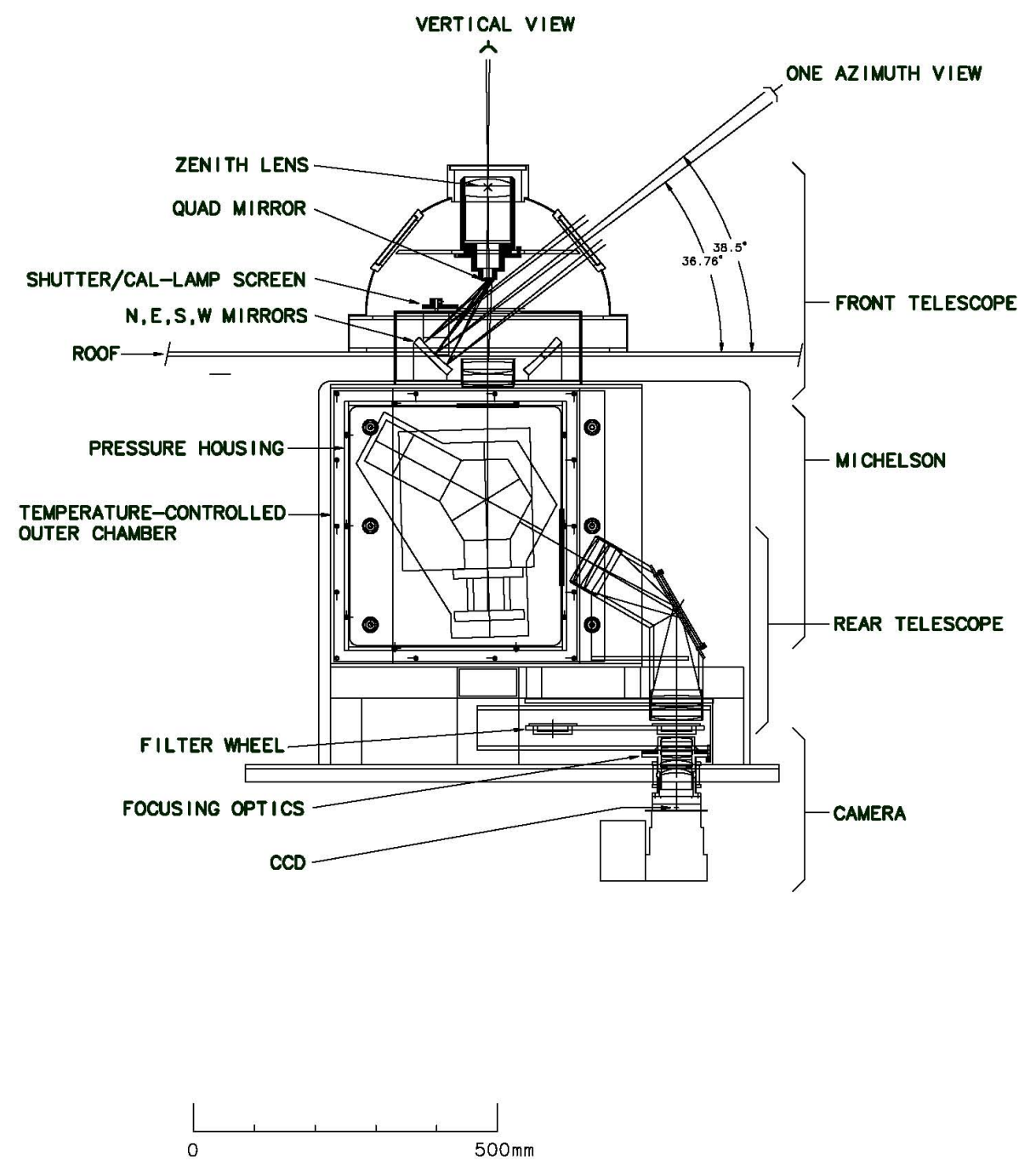

ERWIN-2 MECHANICAL LAYOUT

Fig. 1. Diagram of ERWIN mechanical layout.

combine with the zenith light into a single beam through the rest of the optical system. Each of the cardinal directions views an irregularly shaped piece of the sky approximately 0.0013 steradians at an elevation angle of $38.7^{\circ}$. Zenith is viewed with a solid angle of 0.0007 steradians. Light from each of the cardinal directions is focused onto one of the trapezoidal facets of the quad mirror using spherical mirrors and zenith is focused onto the plane of this mirror. The quad mirror is located at the field stop of the front telescope. The light from these various viewing directions is passed as collimated light through the interferometer and then focused using a second telescope and camera onto the detector. The sky from each direction is thus focused onto different regions of the detector so that each of these five directions is simultaneously measured. The effective field of view of the instrument is the same as that allowed through the interferometer - a beam of $2.5^{\circ}$ half angle.

The camera was built in-house at York University and uses a $512 \times 512$ pixel, back-thinned, low-noise, frametransfer CCD (E2V Technologies CCD57-10) with an active area of $6.656 \times 6.656 \mathrm{~mm}$. The CCD camera is used with $16 \times 16$ binning, resulting in a final image size of $35 \times 32$ bins. It has a quantum efficiency of 0.85 for the $\mathrm{O}\left({ }^{1} \mathrm{~S}\right)$ emission and 0.45 and 0.4 for the $\mathrm{O}_{2}$ and $\mathrm{OH}$ emissions. This is a significant improvement over the photomultiplier tube for which the quantum efficiency was $\sim .14$, .08 and .06 for the same emissions. An additional advantage arises, as noted above, because of the imaging capability; measurements could be made with enhanced visibility over the original instrument. The increased sensitivity allows for a decrease in the exposure times, while still maintaining 


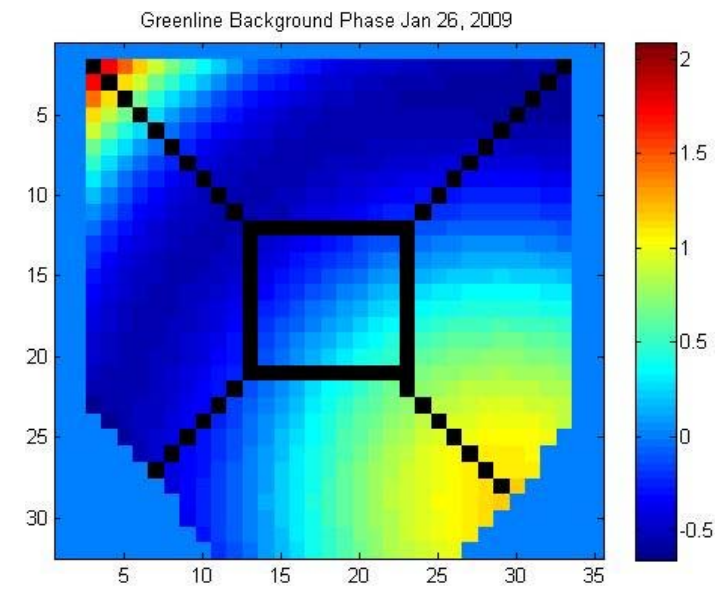

Fig. 2. Green-line background phase (in radians) from 26 January 2009. The $\mathrm{x}$ - and y-axes represent pixel indices. The blackened bins represent the borders between the sections of the quad mirror. The top section measures east, the bottom, west, the left, south, the right, north, and the centre zenith.

comparable signal to noise. For ERWIN II the radiance response is 1.7672 Rayleigh (counts s) ${ }^{-1}$ for green line, 2.0682 Rayleigh (counts s) ${ }^{-1}$ for $\mathrm{O}_{2}$ and 1.0627 Rayleigh (counts $)^{-1}$ for $\mathrm{OH}$.

Figure 2 illustrates the manner in which the field is imaged onto the CCD detector. The dark bins indicate where the edges of the areas illuminated by the various regions of the sky are located. The light from each viewing direction is imaged as follows: the top - east, the bottom - west, the right - north, the left - south, and the centre - zenith. The background colours indicate how the interferometer phase in radians varies across the field and indicate how much variation there is across each region. Variations in phase away from this background along with consideration of the zero wind provide a measure of the Doppler shift in each viewing direction.

The observation procedure of ERWIN II has been updated relative to that described in Gault et al. (1996). Since all viewing directions are now viewed simultaneously, there is no need to cycle through the viewing directions. The basic procedure is to cycle through the three emission measurements in sequence and then after a user specified number of cycles to perform a set of calibrations. A measurement consists of 8 exposures of the detector each at a different value of the Michelson path. The steps (relative to the reference phase which coincides with the initial mirror position) are set to $0,90,180,270,270,180,90$ and $0^{\circ}$ of phase. These steps are different for each emission with $360^{\circ}$ corresponding to a physical change in path of $\lambda$, the wavelength of the emission being viewed. A calibration consists of measurements (again one for each emission) using the calibration lamps and a dark measurement. Initially, a calibration was undertaken after every eight emission scans, but later this was changed to every

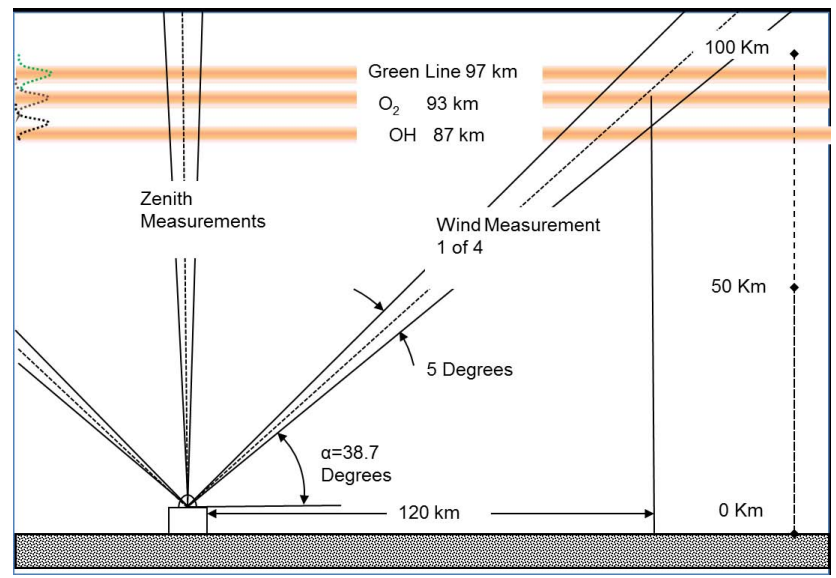

Fig. 3. Schematic of the viewing geometry of ERWIN II. This shows three fields of view. The other two are orthogonal to the plane of the page.

set of emission scans to provide better precision for determining the interferometer drift. Overall, this results in a set of observations (wind determinations for 5 directions and all three emissions) approximately every $3 \mathrm{~min}$. ERWIN takes measurements as long as the solar elevation is less than $0^{\circ}$.

The observing geometry is illustrated in Fig. 3. All three emission layers are shown schematically and occur roughly at nominal heights of 97,93 and $87 \mathrm{~km}$ for the $\mathrm{O}\left({ }^{1} \mathrm{~S}\right), \mathrm{O}_{2}$ and $\mathrm{OH}$ emissions, respectively. Thus, by cycling through the different emissions, information on various heights in the mesopause region is acquired. The azimuthal viewing directions are at an elevation angle of $\alpha=38.7^{\circ}$ from the horizontal. Based on this observing geometry, the $\sim 5^{\circ}$ lateral cross section of each beam, and a mean height of $90 \mathrm{~km}$ and a nominal vertical scale of $\sim 5 \mathrm{~km}$ for the half width of the airglow layer, the volume of atmosphere sampled by ERWIN II is $\sim 5 \mathrm{~km}$ in the vertical and $5 \mathrm{~km}$ by $6 \mathrm{~km}$ in the horizontal. (It will not be sensitive to vertical scales less than approximately half the thickness of the airglow layer $-\sim 5 \mathrm{~km}$ ). Since winds are determined by observing Doppler shifts in the emission frequencies, the observed, line-of-sight winds are combinations of the horizontal and vertical winds (save for the zenith line-of-sight winds, which observe vertical winds).

The meridional wind, $v$, is determined using the difference of the line-of-sight north and south winds

$v=\frac{\left(V_{\mathrm{S}}^{\mathrm{LOS}}-V_{\mathrm{N}}^{\mathrm{LOS}}\right)}{2 \cos \alpha}$,

where

$V_{\mathrm{N}}^{\mathrm{LOS}}=-v \cos \alpha+w \sin \alpha$

$V_{\mathrm{S}}^{\mathrm{LOS}}=v \cos \alpha+w \sin \alpha$.

Here $w$ is the vertical component of the wind and $\alpha$ is the viewing angle of the azimuthal look directions relative to the horizontal $(\cos (\alpha) \approx 0.7804$ and $\sin (\alpha) \approx 0.6252)$. 
Similarly, the zonal wind, $u$, can be determined using the east and west line-of-sight winds,

$u=\frac{\left(V_{\mathrm{W}}^{\mathrm{LOS}}-V_{\mathrm{E}}^{\mathrm{LOS}}\right)}{2 \cos \alpha}$.

The vertical winds can also be obtained using several different approaches. The most precise and accurate is to use the zenith line-of-sight winds

$w=V_{\mathrm{Z}}^{\mathrm{LOS}}$.

The vertical winds can be also be determined indirectly using the cardinal direction line-of-sight winds,

$w=\frac{V_{\mathrm{N}}^{\mathrm{LOS}}+V_{\mathrm{S}}^{\mathrm{LOS}}}{2 \sin \alpha}=\frac{V_{\mathrm{E}}^{\mathrm{LOS}}+V_{\mathrm{W}}^{\mathrm{LOS}}}{2 \sin \alpha}$.

In theory, this provides the means to check the internal consistency of the wind determinations, but only for longer temporal scales. In practice, the effects of gravity waves of scales of the same order as the distance between the viewing points $(\sim 250 \mathrm{~km})$ in the airglow layer will result in oppositely directed fields of view measuring winds that differ as a result of the aliasing of wind variations associated with these waves. This means that these comparisons can only be undertaken for longer term averages for which the spatial scales are significantly larger than $250 \mathrm{~km}$. At the same time these shorter term differences between opposing fields of view allow observations of gravity wave effects to be undertaken, given confidence in the instrument calibration. This will be described in detail in a forthcoming paper.

\section{Data analysis procedure}

The essential issue for wind measurements is distinguishing the phase increments, $\delta \theta$, associated with Doppler shifts in the emission of interest from the phase associated with other factors. In general, the observed phase is a combination of the phase associated with the instrument configuration as manifested for the emission of interest (motionless) plus a shift associated with atmospheric motion of the source region being viewed. In practice, for imaging applications, the phase associated with the instrument configuration can be separated into three terms:

- $\theta_{\mathrm{B}}(i, j):$ a term associated with the phase variation across the field as a function of angle through the interferometer (or pixel location $(i, j)$ on the detector) relative to the phase associated with rays passing at normal incidence through the interferometer (background phase). This variation is illustrated in Fig. 2.

- $\theta_{\mathrm{T}}(t)$ : a term giving the thermal-drift phase of the interferometer relative to the phase observed at a particular time (thermal-drift term).
- $\theta_{0}$ : a term identifying the phase that a motionless source would have (zero-wind phase).

Hence, the measured phase, $\theta_{\mathrm{a}}$, can be expressed as a function of time, $t$, and location on the field as

$\theta_{\mathrm{a}}(i, j, t)=\theta_{\mathrm{B}}(i, j)+\theta_{0}+\theta_{\mathrm{T}}(t)+\delta \theta(t)$.

This formulation of the phase assumes that the background phase stays constant (generally satisfied for carefully maintained interferometers) and temporal variations can be tracked with a single phase parameter, the thermal drift, which is only a function of time.

In theory, the zero-wind phase should be easy to determine since it only requires a stationary source for the emission of interest. In practice, however, this is difficult to achieve since portable sources for the airglow emissions are unavailable and the atmosphere is generally in motion. As with other ground-based wind instruments, a daily average of the observed vertical wind is used to determine this parameter. In the case of ERWIN II this average is measured using the quadrant looking vertical. For measurements at Eureka, where close to $24 \mathrm{~h}$ each day is observed, this determination is expected to be a good measure of the actual zero wind; all periods of tidal motions are covered during this time period, variations associated with gravity waves are expected to average to zero, vertical winds associated with planetary waves are of the order of $\mathrm{cm} \mathrm{s}^{-1}$ and mean vertical winds are small (an ascent or descent of $10 \mathrm{~km} \mathrm{day}^{-1}$ corresponds to a vertical velocity of $0.11 \mathrm{~m} \mathrm{~s}^{-1}$ ).

For ERWIN II the background phase determination was more difficult than expected. Initially, it was thought the phase variation associated with the reference emissions would be suitable to use since they were within a few Ångströms of the emission of interest. However, it was found that use of such a background resulted in phase variations of $\sim 10 \mathrm{~m} \mathrm{~s}^{-1}$ across several of the quadrants when daily averages of the winds were calculated. This effect is thought to be due to differences between the calibration optics and main optics which result in the light distribution across the aperture of each system being different and resulting in a different weighting of any residual path variations in the interferometer. Instead, the background phase which was used was determined using wind observations from a period when it was known to be cloudy (this phase variation is illustrated in Fig. 2 for the green line). Light from the sky was suitably scattered so that all directions gave the same Doppler shift. Daily averages of this wind for the oxygen green-line and hydroxyl observations using this background are presented in Fig. 4. The resulting wind variations are minimal in each quadrant and the mean wind in each quadrant provides a measure of the mean wind for the day. Details of the analysis which lead to this choice of background wind are contained in Kristoffersen (2012).

Although the analysis approach described in Gault et al. (1996) could be implemented for ERWIN II by integrating 
the signal in each quadrant and treating each quadrant as a single detector, a new bin-by-bin least-mean-squares approach similar to that implemented in Ward (1988) was preferred. The latter approach was more precise since it avoids any visibility reduction that occurs as a result of the integration over the phase in each quadrant, and it allows bins contaminated by stars or cosmic ray hits to be eliminated. In addition, a rigorous determination of the wind error can be undertaken.

For this approach, a non-linear least-mean-squares analysis using the Levenberg Marquardt method (as described in Press et al., 2007) is implemented. The variation in photons detected per integration time for a step during the $k$-th 8-point scan is modelled according to the following equation:

$I\left(i, j, t_{k}, s\right)=I_{0}\left(t_{k}\right)\left(1+U V\left(t_{k}\right) \cos \left(\delta \theta\left(t_{k}\right)+\theta_{\mathrm{I}}\left(i, j, t_{k}, s\right)\right)\right)$,

where the parameters being solved for are $I_{0}\left(t_{k}\right)$, the number of photons detected per integration time for a step in the absence of interference, $U V\left(t_{k}\right)$, the net visibility, and $\delta \theta\left(t_{k}\right)$, the phase variation associated with the wind. Here $t_{k}$ is the time of the $k$-th scan. To ensure that the variation in angles being determined is small, all the known phases associated with a particular bin are added together so that $\theta_{\mathrm{I}}\left(i, j, t_{k}, s\right)=\theta_{\mathrm{B}}(i, j)+\theta_{0}+\theta_{\mathrm{T}}\left(t_{k}\right)+\left(\Delta \theta_{\mathrm{s}}\right)$, where $\left(\Delta \theta_{\mathrm{S}}\right)$ is the step size increment associated with mirror step of index s (expressed as a phase angle).

The merit function is

$\chi^{2}=\sum_{s=1}^{N} \frac{\left(\overline{I_{\mathrm{s}}}\left(i, j, t_{k}, s\right)-y\left(I\left(i, j, t_{k}, s\right) ; a_{l}\right)\right)^{2}}{\sigma_{\mathrm{s}}^{2}\left(i, j, t_{k}, s\right)}$,

where

$y\left(I\left(i, j, t_{k}, s\right) ; \quad a_{l}\right)=a_{l}(1)\left(1+a_{l}(2) \cos \left(a_{l}(3)+\theta_{\mathrm{I}}\left(i, j, t_{k}, s\right)\right)\right)$

$a_{l}=\left(I_{0_{l}}\left(t_{k}\right), \quad U V_{l}\left(t_{k}\right), \quad \delta \theta_{l}\left(t_{k}\right)\right)$.

$\overline{I_{\mathrm{S}}}\left(i, j, t_{k}, s\right)$ is the number of photons detected for steps $s$, bin $(i, j)$ and scan $k$, and $\sigma_{\mathrm{s}}^{2}\left(i, j, t_{k}, s\right)$ is the associated variance (estimated as shot noise). $l$ is the iteration index, so $y\left(I\left(i, j, t_{k}, s\right) ; a_{l}\right)$ is the number of photons detected determined using the constants determined on the $l$-th iteration. $\theta_{\mathrm{I}}\left(i, j, t_{k}, s\right)$ with $\Delta \theta_{\mathrm{S}}=0$ is used to seed the first iteration of this method. Typically, only a few iterations are needed for convergence to a solution.

In practice, there are two approaches used to determine the zero wind and thermal-drift phase: one using the calibration lamp phase determinations (the standard procedure) and the other when the calibration lamp phase is not available (as occurred for the $\mathrm{OH}$ emission when the lamp malfunctioned). For the standard procedure, the calibration phase was determined on a regular basis throughout the night by calculating the average Michelson phase over the full field of the calibration lamp using the same 8-point scan as for the airglow observations. The resulting time series of calibration phase is interpolated using cubic splines to the airglow observation times to provide the variation in the thermal-drift phase. The relative airglow phase throughout the observation period is then calculated using the non-linear approach with the zerowind phase set to zero. The actual zero-wind phase then provides an offset to this relative phase and is determined using the mean of the zenith over the entire day. The winds are determined by shifting the relative airglow phase by the zerowind phase and using Eq. (2) to convert the phase to velocity.

If the calibration lamps are not available, then the zenith measurements can be used to estimate the phase associated with the thermal drift and zero wind. In this case, the analysis approach as described above is undertaken but with the thermal-drift and zero-wind terms set to zero. For each image, the phase for the vertical view is then taken as an estimate of the sum of these two terms and subtracted off the four other quadrants. The disadvantage of this approach is that the vertical wind cannot be determined and phase variations associated with vertical winds are mixed into the phase determinations for each of the cardinal directions. This reduces the precision and accuracy of the radial wind determination. Nevertheless, the meridional and zonal wind determinations are unaffected by the lack of an independent zero-wind determination since they are determined through a difference in radial wind determinations in opposite directions, and hence the zero-wind contribution to the two directions is eliminated (see Eqs. 5 and 8).

There are several advantages associated with the standard approach described above. This algorithm can be run for fewer than the 8 steps comprising a typical scan. Because the steps are in phase multiples of $90^{\circ}$ and all phases steps are repeated twice, anomalous numbers of photons detected at a particular step can be identified by comparing photon numbers observed for steps of the same phase and examining the sums of photon numbers for steps which are $180^{\circ}$ apart. If one or two steps with anomalous numbers of detected photons are identified in a scan, they are eliminated from the phase determination, thereby reducing the likelihood of outliers. In addition, there are $\sim 150$ bins used for wind determinations in each of the cardinal directions and $\sim 80$ bins for the vertical. As a result, any additional outliers beyond three standard deviations of the mean wind phase in each of the quadrants can be identified and are then eliminated and the standard error associated with the wind determination for each quadrant calculated. Implementation of these checks results in a robust statistical framework for the wind determinations with this instrument.

\section{Measurement validation}

In this section, results which verify the precision and accuracy of the ERWIN II instrument are presented. Crucial is an accurate determination of the background phase variation across the field, the thermal drift and the zero wind. Each of these aspects of the wind determination contributes to the 

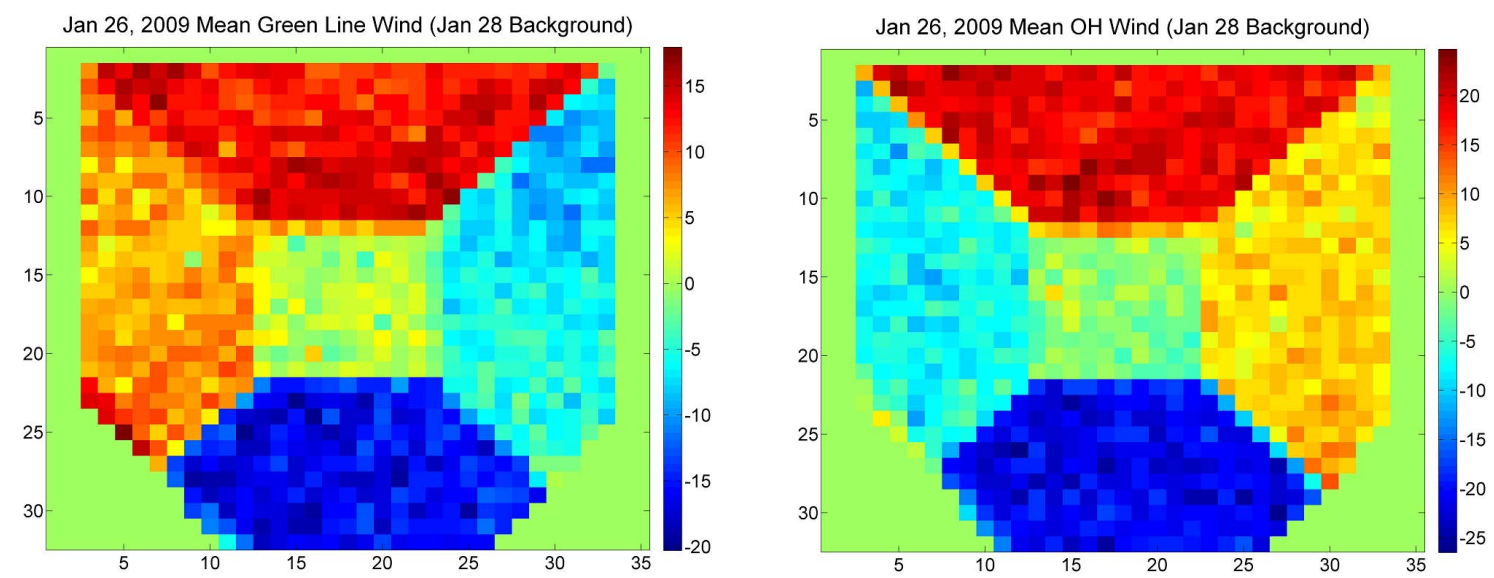

Fig. 4. An image of the daily averaged wind image $\left(\mathrm{m} \mathrm{s}^{-1}\right)$ for the green-line and OH observations on 26 January 2009 , using the 28 January 2009 phase average for the background phase. The averages are calculated on a bin-by-bin basis. The sector averages of these images provide the daily mean winds in each direction. The uniformity of each sector and the fact that opposite sectors are of similar magnitude but opposite sign indicates that the phase background is not introducing systematic errors into the wind determinations.

measurement precision and accuracy. Evaluation of the measurement quality in terms of these aspects is possible because of redundancies in the approach and tests which were undertaken to investigate specific aspects of the measurement approach.

As noted above, the background phase was determined using observations taken on 28 January 2009 during a period when it was cloudy so that the directional asymmetry in the Doppler shifts would be negligible as a result of scattering in the clouds. During the eight hours of observations used to determine this phase variation, $\sim 160$ measurements were taken. The background phase was determined by taking the average of these measurements. For the fringe parameters associated with these measurements, the standard deviation of the wind determination for each bin on each scan was $\sim 15 \mathrm{~m} \mathrm{~s}^{-1}$ so that would be the standard error for the background phase determination was $\sim 1.1 \mathrm{~m} \mathrm{~s}^{-1}$ for each bin. Since winds are determined by averaging the phases determined on a bin-by-bin basis in each sector, this error makes a negligible contribution to the measurement precision.

Equally important is determining whether there are any systematic errors in the background determination. This is important since the winds in opposite directions are determined relative to the background phase so errors in the background phase would result in systematic errors in these winds. Figure 4 shows the daily average wind image measured on 26 January 2009 for the oxygen green-line and hydroxyl observations. Shown for each emission is the daily average of each bin in the image. The average of each sector gives the mean radial wind in each direction for this day. Two things of particular note are the uniformity of the winds in each quadrant and that opposite quadrants are close to the same magnitude but oppositely signed. The uniformity indicates that the background phase has been effectively removed from the wind determinations since the gradient associated with the angular dependence of the background phase is not observed. The fact that the winds in opposing directions are consistent with what would be expected geometrically indicates that errors in the background phase determination are minimal and do not result in systematic errors in the radial wind determinations. The wind is defined as positive towards the instrument, so the observation of a positive (negative) wind in one sector would correspond to a wind of the opposite sign in the opposite quadrant.

This self-consistency of ERWIN II is further demonstrated by considering the averages of these sectors for several days, shown in Table 1. Since the winds in opposing directions should have the same magnitude but opposite sign, the sum of these values should be zero. The table provides the values of the average daily phase for each of the four sectors corresponding to the cardinal directions for three days in January 2009 for the hydroxyl and green-line observations. The means for each sector over the three days is calculated (4th column) and then the sum of the phases of opposite sectors determined. Since the winds in opposing directions should have the same magnitude, but opposite sign, the sum of these values should be close to zero, as observed. On average, the difference is of the order of $1 \mathrm{~m} \mathrm{~s}^{-1}$. This further validates that the background is suitable for the wind determinations and at most a systematic error of $1 \mathrm{~m} \mathrm{~s}^{-1}$ is introduced into the radial wind observations. The use of this phase background has been checked for the subsequent years and it is a stable feature of the interferometer.

These results also demonstrate that, on average, the thermal drift is being calculated properly. Since the thermal drift would be a constant value added to the phase for every bin, at a given time, this value would have the same sign for each section. This would result in an addition of the thermaldrift terms, rather than the cancellation of the winds, as is observed. 
Table 1. Comparisons of the mean differences of the daily averages of the opposite sight directions for the green-line and OH emissions using the 28 January 2009 background phase.

\begin{tabular}{|c|c|c|c|c|c|c|}
\hline & \multicolumn{6}{|c|}{ Green line - 28 January background } \\
\hline & \multicolumn{5}{|c|}{ Mean phase (degrees) } & \multirow{2}{*}{$\begin{array}{r}\text { Difference } \\
\quad\left(\mathrm{m} \mathrm{s}^{-1}\right)\end{array}$} \\
\hline & $23 \mathrm{Jan}$ & $25 \mathrm{Jan}$ & $26 \mathrm{Jan}$ & Mean & Difference & \\
\hline Bottom - West & -2.2689 & -5.2311 & -4.3201 & -3.9419 & -0.3266 & -1.4216 \\
\hline Top - East & 2.183 & 5.5978 & 3.0596 & 3.6154 & & \\
\hline Left - South & -2.3606 & -0.424 & 1.2777 & -0.5042 & -0.0115 & -0.0416 \\
\hline \multirow[t]{4}{*}{ Right - North } & 3.5065 & -0.1719 & -1.8564 & 0.4927 & & \\
\hline & \multicolumn{6}{|c|}{$\mathrm{OH}$ - 28 January background } \\
\hline & \multicolumn{5}{|c|}{ Mean phase (degrees) } & Difference \\
\hline & $23 \mathrm{Jan}$ & $25 \mathrm{Jan}$ & $26 \mathrm{Jan}$ & Mean & Difference & $\left(\mathrm{m} \mathrm{s}^{-1}\right)$ \\
\hline Bottom - West & -1.885 & -2.6528 & -2.9221 & -2.4866 & 0.1948 & 0.8563 \\
\hline Top - East & 1.9366 & 3.4263 & 2.6872 & 2.6814 & & \\
\hline Left - South & -2.5153 & -2.7731 & -0.8938 & -2.0626 & 0.2177 & 0.9477 \\
\hline Right - North & 3.4492 & 2.3319 & 1.0542 & 2.2804 & & \\
\hline
\end{tabular}
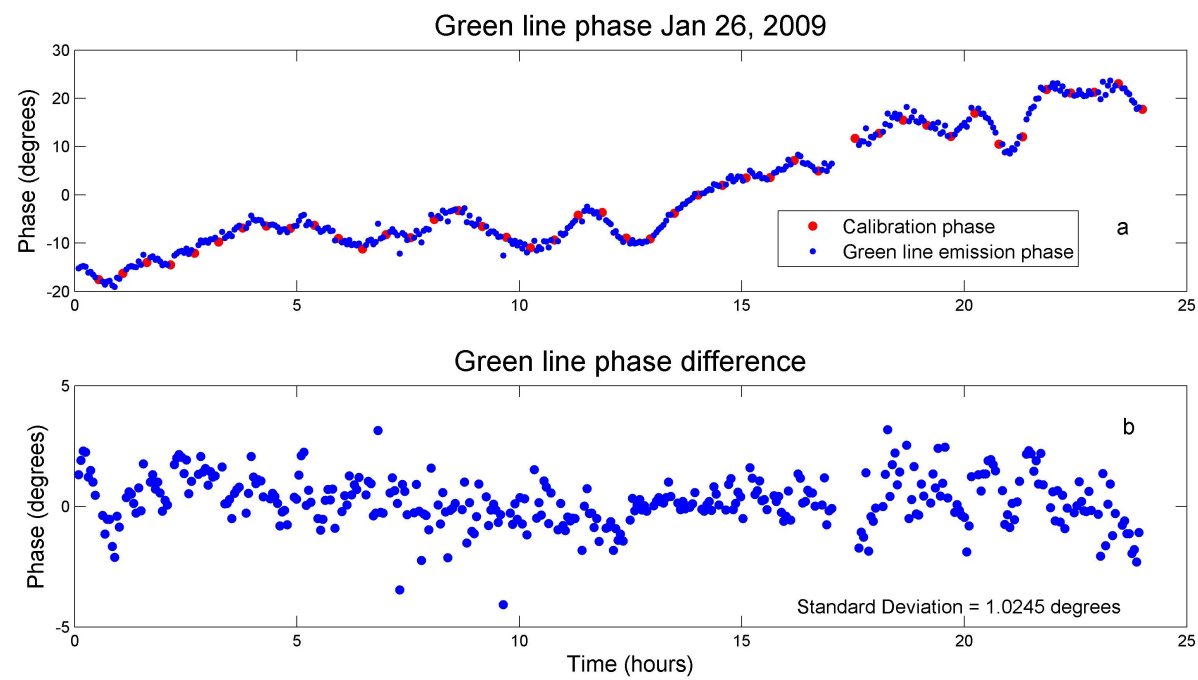

Fig. 5. (a) Plot of green-line-emission zenith phase (blue dots) and the calibration phase (red dots) on 26 January 2009 . The measurement uncertainty of the phase measurements is smaller than the size of the dots in this plot. (b) Plot of the difference between the zenith phase and the calibration phase interpolated using cubic splines to the times of the atmospheric observations. The zenith observations include geophysical variability. The time is UTC.

Depending on the thermal stability of the instrument shortterm measurement errors can also be introduced if the thermal drift is not followed with sufficient precision. As described earlier, initially calibration phase measurements were taken on a slower cadence than the measurements. For the first two years of observations, one scan of the calibration lamps was taken for every eight scans of the atmospheric emissions. For the third year (March 2010 to March 2011) this was increased to a calibration every measurement scan of the atmospheric emissions.
Figure 5 gives an indication of the precision of the thermal-drift determination. Figure 5a (upper panel) shows a time series of the green-line zenith phase (blue points) along with the phase determined with the calibration lamp (red dots - corrected with the zero-wind offset so that both time series have the same daily average). On this figure the uncertainties in the phase determinations are smaller than the diameter of the dots in the plots. The two phase determinations follow each other closely. 
Figure $5 \mathrm{~b}$ (lower panel) is a time series of the difference between the observed zenith phase and the calibration phase interpolated using cubic splines to the times of the atmospheric observations. In this figure the measurement precision is close to the size of the dots. The standard deviation of the difference is $1.02^{\circ}$ or $4.5 \mathrm{~m} \mathrm{~s}^{-1}$. This number includes geophysical variability (vertical winds, volume emission rate variations) and Schott noise and hence is not a clean measure of the uncertainty introduced through the thermal-drift calibrations.

To explore this aspect of the measurement in more detail, an experiment which included frequent calibration measurements was performed (a calibration measurement was taken after every atmospheric measurement). The resulting calibration phase time series was compared to one constructed by sampling this time series at a the same rate as other typical days and then interpolating (using cubic splines) these measurements to the times of the original time series. This process duplicated the thermal phase determinations associated with the atmospheric measurements

Figure 6 shows the result of this experiment. The phase error in the cubic spline is never more than a degree, and the standard deviation of the phase error over the duration of this experiment is $0.328^{\circ}\left(1.43 \mathrm{~m} \mathrm{~s}^{-1}\right)$. This is significantly smaller than the standard deviation of the difference of the calibration phase and the zenith emission phase, which was $1.02^{\circ}\left(4.5 \mathrm{~m} \mathrm{~s}^{-1}\right)$. This demonstrates that the errors associated with the cubic spline are acceptably small compared to the other errors and geophysical noise. This uncertainty is reduced for observations with the more frequent calibration cadence.

Using the value of " $a$ " appropriate for the 8-step scan, and substituting appropriately in Eqs. (2) and (4), the following expression for the standard deviation (which we use as a measure of the wind precision) is obtained:

$\sigma_{\mathrm{w}}=\frac{c \lambda}{4 \pi \Delta_{\mathrm{eff}} U V \sqrt{I}}$

Here $\sigma_{\mathrm{w}}$ is the error in $\mathrm{m} \mathrm{s}^{-1}, \Delta_{\text {eff }}$ is the effective path difference, $U V$ is the visibility, $I$ is the observed number of photons detected per integration time per step in the absence of interference effects, $c$ is the speed of light and $\lambda$ is the wavelength of the emission. The wind standard deviation is inversely proportional to $U V$, the path difference and the square root of $I$. Comparison of the estimated variance using this formula to the observed variance provides an indication of how closely the observations conditions during individual scans match the assumptions associated with the derivation of this formula (namely that the source radiance and visibility remain constant during a scan).

This is illustrated in Fig. 7, which provides time series of the actual standard error and the standard error estimated using Eq. (12) (calculated as a variance for each bin in the sector using Eq. (14) and then averaged in the same way as the

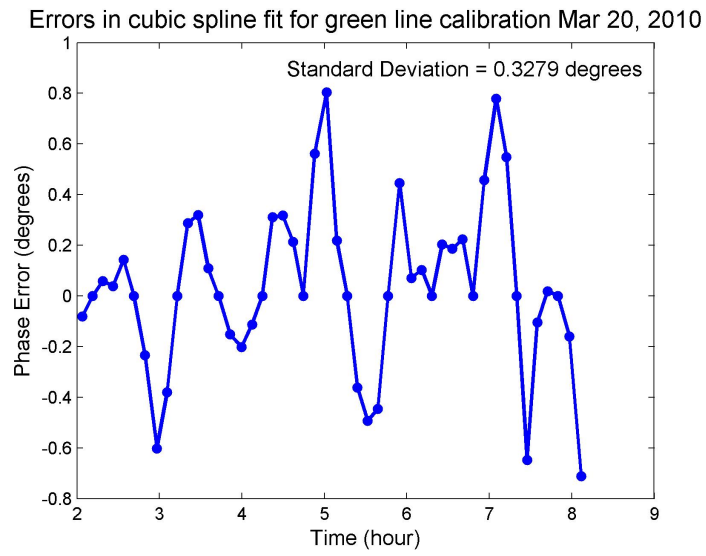

Fig. 6. Plot of the error in the cubic spline interpolation for the green-line calibration lamp sampled every $\sim 30$ min relative to the original time series and interpolated to the times of the original time series. Every 8th point of the original time series is used for the interpolation (the error for these points is identically zero). The data were recorded on 20 March 2010.

actual standard error is calculated), the signal (in analogue to digital units - ADU) and visibility for the north sector on 22 December 2008. The variability in both data sets is due to variations in the airglow volume emission rate (primarily) and visibility during that day. The expected decrease in the standard error when the signal increases (and vice versa) is evident in the upper two plots. It is striking that the two measures follow each other so closely since this indicates that intensity and visibility variations during individual scans are generally insignificant. If they are a factor then the standard error would be significantly larger than the estimated precision.

Figure 8 shows time series of the standard error for all four cardinal direction sectors for 26 January 2009. For this date, some twilight was present in the afternoon centred at $\sim$ 17:00 UTC. The increase in the standard error in the vicinity of this time is the expected result of increases in the background relative to the airglow volume emission rate (see discussion at the end of Sect. 2). The time series of all four sectors follow each other well. The daily average of the greenline standard error for the zenith section is $1.91 \mathrm{~m} \mathrm{~s}^{-1}$, and that for all of the cardinal directions is $1.22 \mathrm{~m} \mathrm{~s}^{-1}$. The daily average of the precision based on the visibility and intensity of the green-line emission for zenith is $1.88 \mathrm{~m} \mathrm{~s}^{-1}$, and that for the cardinal directions is $1.24 \mathrm{~m} \mathrm{~s}^{-1}$. The values for zenith are greater than for the cardinal directions because of geometrical considerations. The effective layer thickness (and hence irradiance) is greater by $1 / \cos (\alpha)$, where $\alpha$ is the inclination angle relative to zenith (here $\alpha=51.3^{\circ}$ ). The irradiance during this day was lower on average, so these values are slightly higher than the typical precision of these measurements. 

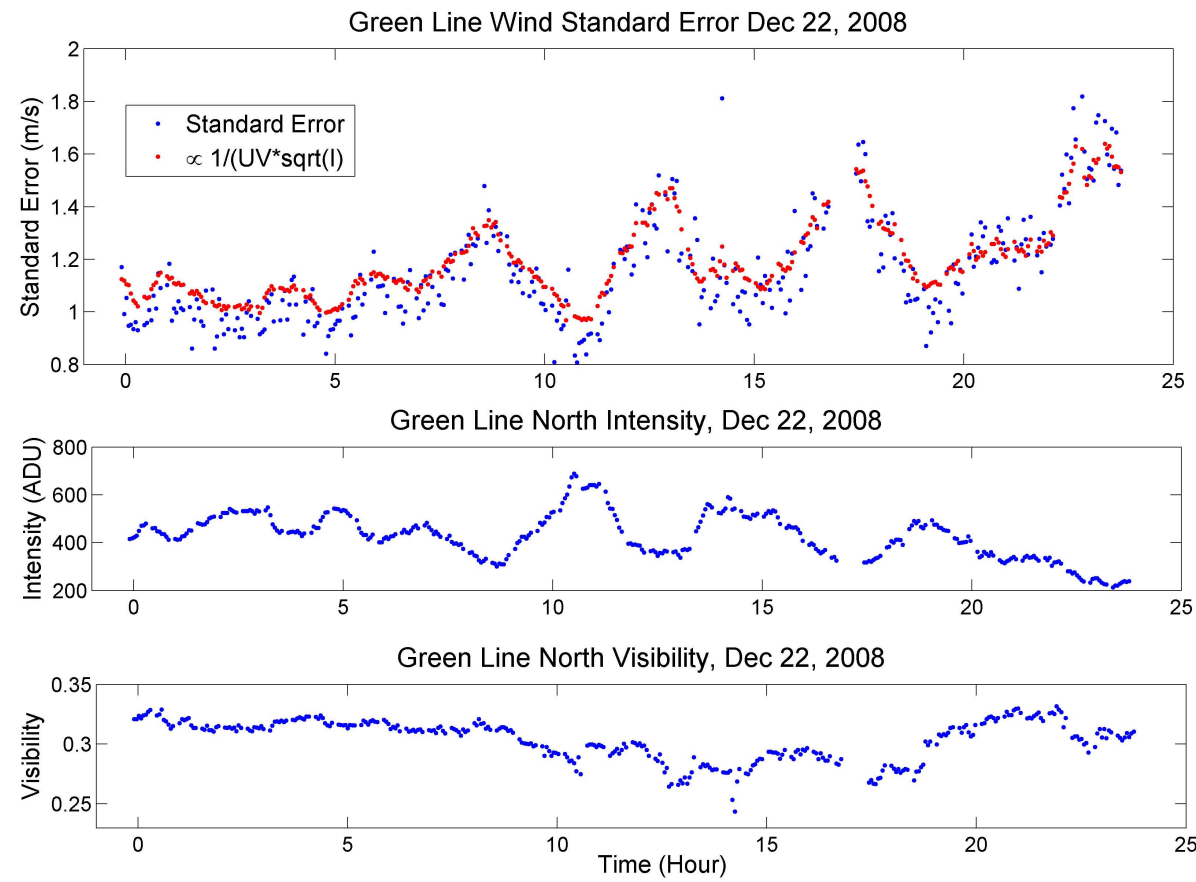

Fig. 7. Comparison of the standard error (blue points) for northern sector compared to the $\sigma_{\mathrm{W}}$ (red points). Below this panel the corresponding detected signal (in ADU, middle panel) and visibility (lower panel) as a function of time for the same day are shown. Decreases in the standard error are mainly associated with increases in the signal as expected given the visibility stays roughly constant throughout the day.

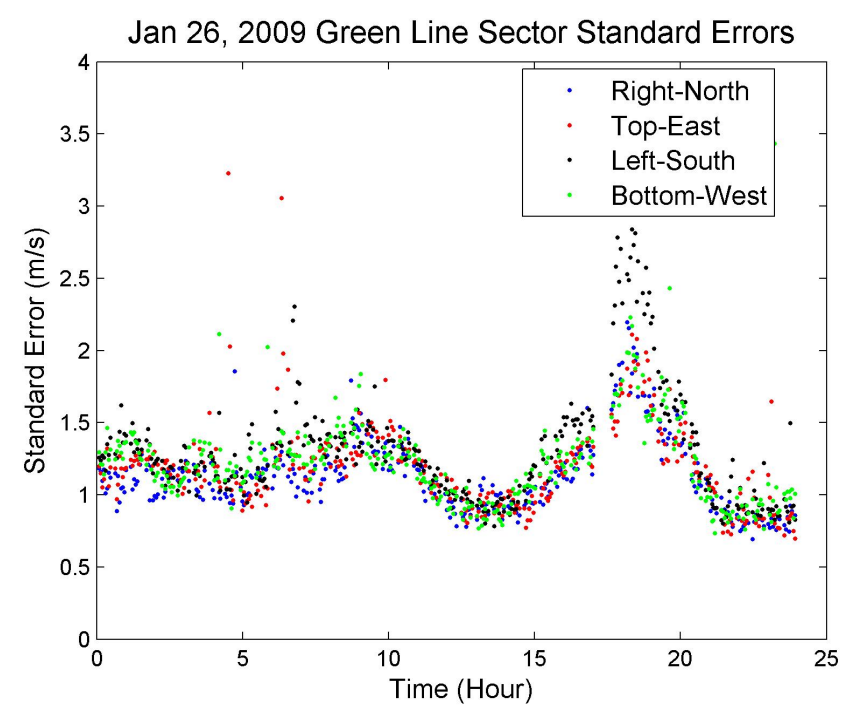

Fig. 8. Standard errors for each of the cardinal direction sectors of the CCD on from 26 January 2009.

Plots of the winds provide a final, and satisfactory, check that the results are accurate. Meridional winds from 23 January 2009 for green line and hydroxyl calculated in several different ways are shown in Fig. 9. The meridional, north direction and south direction winds are calculated using Eqs. (5)-(7), respectively. The time series designated "without subtracting zenith" is a time series of the wind determined from the northern sector without zenith subtracted. The meridional winds are reasonable, and indicate that there was a significant semi-diurnal tidal variation present. The manner in which this wind is determined renders it independent of vertical wind and thermal drift. Although perturbations due to gravity waves will result in small deviations from the true large-scale meridional wind above the station, to good approximation this time series can be considered a good measure of the true meridional wind. Issues with thermal-drift, zero-wind or background phase will result in the meridional winds determined solely from the north or south directions deviating significantly from this time series.

All the green-line winds agree well with each other. The north and south winds, which are the winds determined from the respective sectors with the zenith phase subtracted off to remove any thermal drift, follow the meridional wind very closely. This demonstrates that the winds viewed from the different directions are self-consistent. The fourth wind plotted on this figure is the north wind without the zenith phase subtracted. Since this also fits the meridional wind very closely, it demonstrates that the thermal drift has been effectively removed from the wind phase for observations with this emission.

The $\mathrm{OH}$ observations are taken during the time when the associated calibration lamp was not working. As with the green-line observations, the north and south winds follow the meridional wind closely. In contrast, the north wind without zenith subtracted deviates significantly from the other winds. 

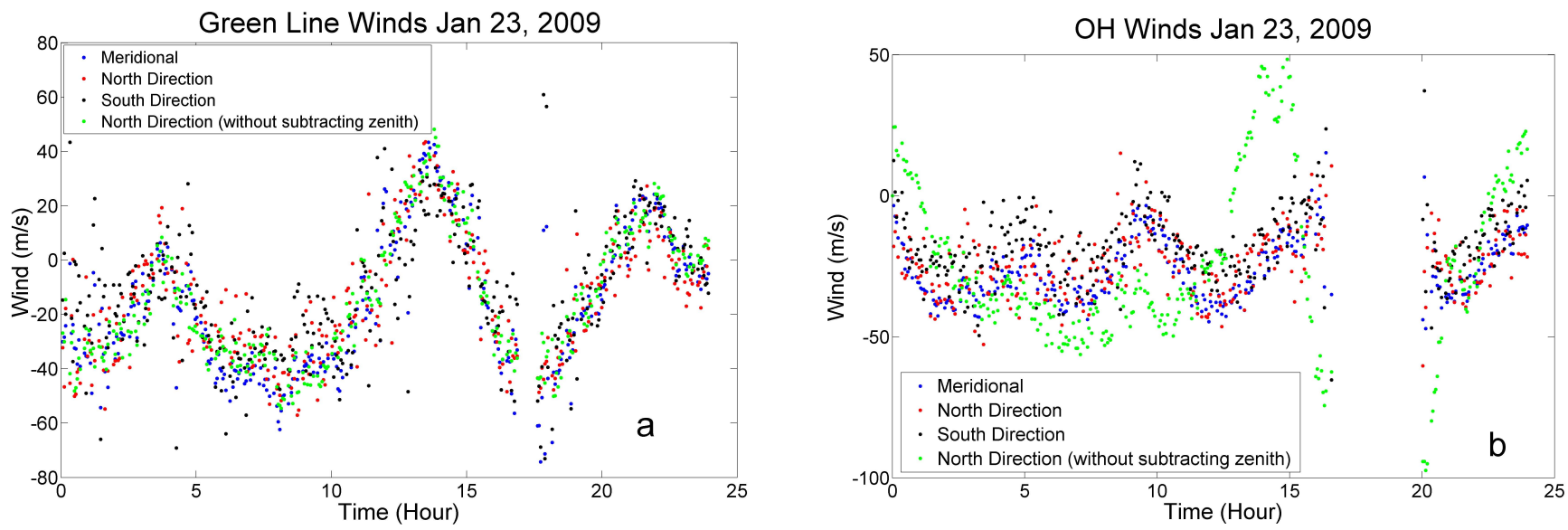

Fig. 9. Time series of meridional winds $\left(\mathrm{m} \mathrm{s}^{-1}\right)$ from 23 January 2009 for the green-line (a) and hydroxyl emission (b) observations showing the consistency of the observations in the north and south directions when thermal drift and zero wind are appropriately accounted for and the problems - green points - in (b) when they are not. The standard errors of these observations are approximately the size of the points in the figure. Detailed discussion is in the text.

As expected, since there are no calibration measurements to provide thermal-drift information, omission of the zenith phase results in winds which exhibit significant systematic errors.

Since the contribution of the thermal-drift variance to the wind observations is $\sim 2.85 \mathrm{~m}^{2} \mathrm{~s}^{-2}$ for the time when the longer cadence calibration period was implemented, it is possible to use the zenith phase to examine the vertical wind (for the shorter cadence calibration period this will be even more feasible). While the exploration of this possibility will require careful analysis, an indication that this is plausible comes from a comparison (see Fig. 10) between the vertical wind determined using the zenith phase (blue dots) and the vertical wind determined using Eq. (10) (black dots). Both of these methods provide similar results on the larger temporal scales. Over the day, the vertical wind is modulated sinusoidally by a few $\mathrm{m} \mathrm{s}^{-1}$. This could be due to a diurnal tide, although since this day was during the 2009 major stratospheric warming, other dynamical effects could be present (Manney et al., 2009). The variance in the directly measured vertical wind is $30.9 \mathrm{~m}^{2} \mathrm{~s}^{-2}$, which is significantly greater than the contribution associated with the thermal-drift determination. The variance in the indirect determination of the vertical wind is $89.4 \mathrm{~m}^{2} \mathrm{~s}^{-2}$. This calculation however also includes contributions from horizontal motions due to gravity waves and other high wave number phenomena. Further analysis of the vertical winds will be undertaken in the future to determine whether definitive geophysical results are possible.

In this section, the various factors affecting the precision and accuracy of the ERWIN II wind results have been discussed and results demonstrating the internal consistency of the winds presented. The main factors affecting the measurement precision are the uncertainties associated with

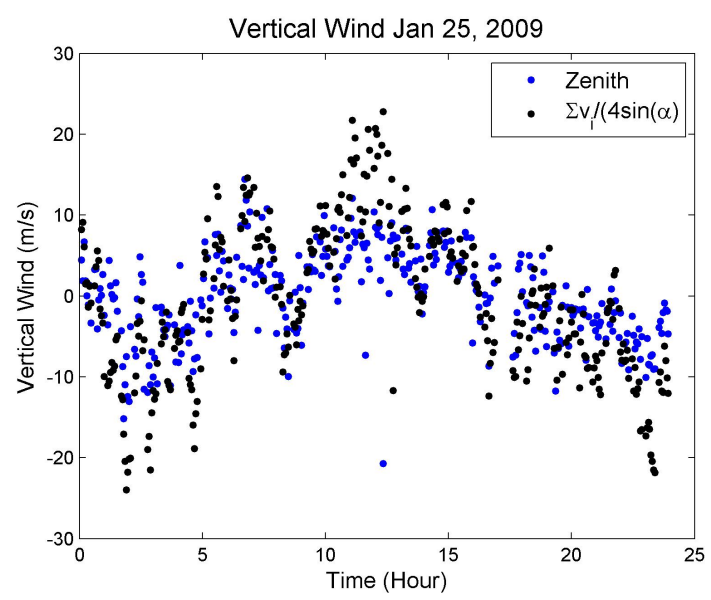

Fig. 10. Green-line vertical wind $\left(\mathrm{m} \mathrm{s}^{-1}\right) 25$ January 2009 as directly observed (blue dots) and as calculated using the north, south, east and west winds (black dots) according to Eq. (10).

the Schott noise and the thermal-drift determination. For the observations with the low cadence calibration measurements the net variance is $\sim 4 \mathrm{~m}^{2} \mathrm{~s}^{-2}$ (i.e. $\left.(1.22)^{2}+(1.43)^{2}\right)$ for the green-line and $\mathrm{OH}$ observations and $\sim 19 \mathrm{~m}^{2} \mathrm{~s}^{-2}$ (i.e. $\left.(4.0)^{2}+(1.43)^{2}\right)$ for the $\mathrm{O}_{2}$ observations. These values depend on the manner in which the thermal drift varies and for the observations taken with the high cadence calibrations will be close to the Schott noise values alone. The $\mathrm{O}_{2}$ values are significantly greater than the other two emissions because its irradiance is significantly smaller. For all the emissions, the precision is dependent on the detected number of photons/step and visibility. There are occasions when the airglow volume emission rate is close to a factor of four greater than that for the days used in this paper to validate the instrument. 
On these days the standard error will be less than $1 \mathrm{~m} \mathrm{~s}^{-1}$ for observations during which the calibration cadence was high.

The measurement accuracy for radial winds is determined by the uncertainty in the background phase determination $\left(<1 \mathrm{~m} \mathrm{~s}^{-1}\right)$ and uncertainties in the zero-wind determination. Based on the arguments presented in Sect. 4, this is expected to be less that $1 \mathrm{~m} \mathrm{~s}^{-1}$ also. Hence, for the best observation procedure (high cadence calibrations), the precision and accuracy are both $\sim 1 \mathrm{~m} \mathrm{~s}^{-1}$.

\section{Discussion}

Comparisons between the capabilities of ERWIN II and other optical wind measuring instruments are not straightforward. Although theoretical comparisons based on throughput considerations have been undertaken (see Shepherd, 2002), few papers have discussed the precision and accuracy of a technique in practice in as much detail and as clearly as has been undertaken in this paper. In part this is because a standard for comparison has not been developed, in part because the precision is dependent on the integration time (i.e. amount of light collected), instrument aperture and field of view, and thermal stability, and lastly in part because clear identification of the zero wind is difficult in practice. Instead, estimates of the measurement precision tend to be embedded in measurement papers using the particular technique in question. Since most techniques use some sort of average of the vertical wind over a night to estimate the true zero, unless there are other systematic errors, one can assume that the measurement accuracies are similar. As a result the measurement precision (taken as the standard deviation of the velocity determination) and the integration time needed for the measurement as quoted in the literature are the only pragmatic means to use in comparing different instruments.

For ERWIN II, five velocity measurements at a precision of $1 \mathrm{~m} \mathrm{~s}^{-1}$ in $45 \mathrm{~s}$ are observed for an airglow brightness slightly below average. In a recent paper on multiple order Fabry-Perot wind observations (Shiokawa et al., 2012) (similar to the observation technique used by Makela et al., 2011 and Meriwether et al., 2011) random errors ranging from 2 to $13 \mathrm{~m} \mathrm{~s}^{-1}$ are quoted for a single wind observation with an exposure time of $60 \mathrm{~s}$. Assuming that observing conditions in the middle of this range correspond to those for ERWIN II, it would take this instrument $\sim 5 \mathrm{~min}$ to observe the same five velocities with a precision of $\sim 7 \mathrm{~m} \mathrm{~s}^{-1}$.

The Scanning Doppler Interferometer (SCANDI) described by Arulia et al. (2010) is used for all-sky auroral imaging by using multiple fringes. They note that it takes $\sim 7-8$ min to obtain a 25 -sector wind measurement for these emissions, which are about an order of magnitude greater in brightness than they are at mid-latitudes (i.e. airglow as with ERWIN-II). For measurements on 8 March 2007 an uncertainty of $15 \mathrm{~m} \mathrm{~s}^{-1}$ is quoted. Assuming that the measurement uncertainty scales roughly as the reciprocal of the square root of the brightness, the uncertainty in these measurements would be $\sim 45 \mathrm{~m} \mathrm{~s}^{-1}$ (i.e. $15 \mathrm{~m} \mathrm{~s}^{-1} \times \sqrt{ } 10$ ) for a brightness an order of magnitude less than that observed. If this was reduced to a 5-sector measurement by combining the observed irradiances, then the precision would be $\sim 20 \mathrm{~m} \mathrm{~s}^{-1}$ (i.e. $45 / \sqrt{ } 5$ ) for a $\sim 7 \mathrm{~min}$ measurement.

The DASH instrument (Englert et al., 2007) was recently used in field measurements at a mid-latitude site to compare results to a Fabry-Perot interferometer (Englert et al., 2012). For this comparison, 5 min integrations were taken and the oxygen red line was observed. Uncertainties at the one sigma level ranged from $\sim 5$ to $15 \mathrm{~m} \mathrm{~s}^{-1}$ based on the plots in this paper. It would take $>25 \mathrm{~min}$ to achieve the 5-measurement cycle of ERWIN II at a precision $\geq 5 \mathrm{~m} \mathrm{~s}^{-1}$.

This comparison indicates that the precision and measurement cadence achieved by ERWIN II is significantly superior to any others reported in the literature. Of these, the multiple order Fabry-Perot of Shiokawa et al. (2012) comes closest to the ERWIN II performance. Even with this instrument (again assuming the uncertainty scales with number of photons detected per step as described above) it would require an integration time of $225 \mathrm{~min}\left(5 \mathrm{~min} \times 7^{2}\right)$ to achieve the $1 \mathrm{~m} \mathrm{~s}^{-1}$ precision that ERWIN II achieves. These comparisons are not definitive since the instruments described in these papers do not necessarily represent their optimal configuration. However, the advantage shown by ERWIN II is unlikely to be matched by minor changes in the configurations of these instruments. At this time it provides the most precise and rapid airglow wind measurements in the world.

Rockets, lidar and radar are three other techniques used to measure winds in the mesopause region. Although comparisons with the precision of the wind measurements associated with these techniques are undertaken below, other important aspects of the dynamical fields are measured using these instruments (for example temperature and density with lidar and diffusion with rockets and radar). In addition, their measurements may be more extensive that those undertaken with ERWIN II (i.e. inclusion of day/night observations or a greater height range and vertical resolution).

Rockets provide short-term wind observations in this region with a wind precision of $5-10 \mathrm{~m} \mathrm{~s}^{-1}$, a temporal resolution of $3 \mathrm{~s}$ and a height resolution of $\sim 1 \mathrm{~km}$ over about 10 min (Larson, 2002; Chu et al., 2007 and references therein). She et al. (2003) state that their sodium lidar has a vertical resolution of $2 \mathrm{~km}$ and measures winds between 81 and $107 \mathrm{~km}$. The wind precision is $\sim 1.5 \mathrm{~m} \mathrm{~s}^{-1}$ at the peak of the layer $(\sim 91 \mathrm{~km})$ and $\sim 15 \mathrm{~m} \mathrm{~s}^{-1}$ at the upper and lower bounds of their measurements for a $1 \mathrm{~h}$ integration during night. In a comparison between radar (middle and upper atmosphere - MU - radar (meteor mode)) and Fabry-Perot winds observed over Shigaraki, Japan, Fujii et al. (2004) quote uncertainties of $2-5 \mathrm{~m} \mathrm{~s}^{-1}$ for $1 \mathrm{~km}$ resolution and $30 \mathrm{~min}$ integrations at $\sim 93 \mathrm{~km}$, which is close to the height of maximum meteor trail detection. Radial wind uncertainties associated with a meteor radar in a paper by 

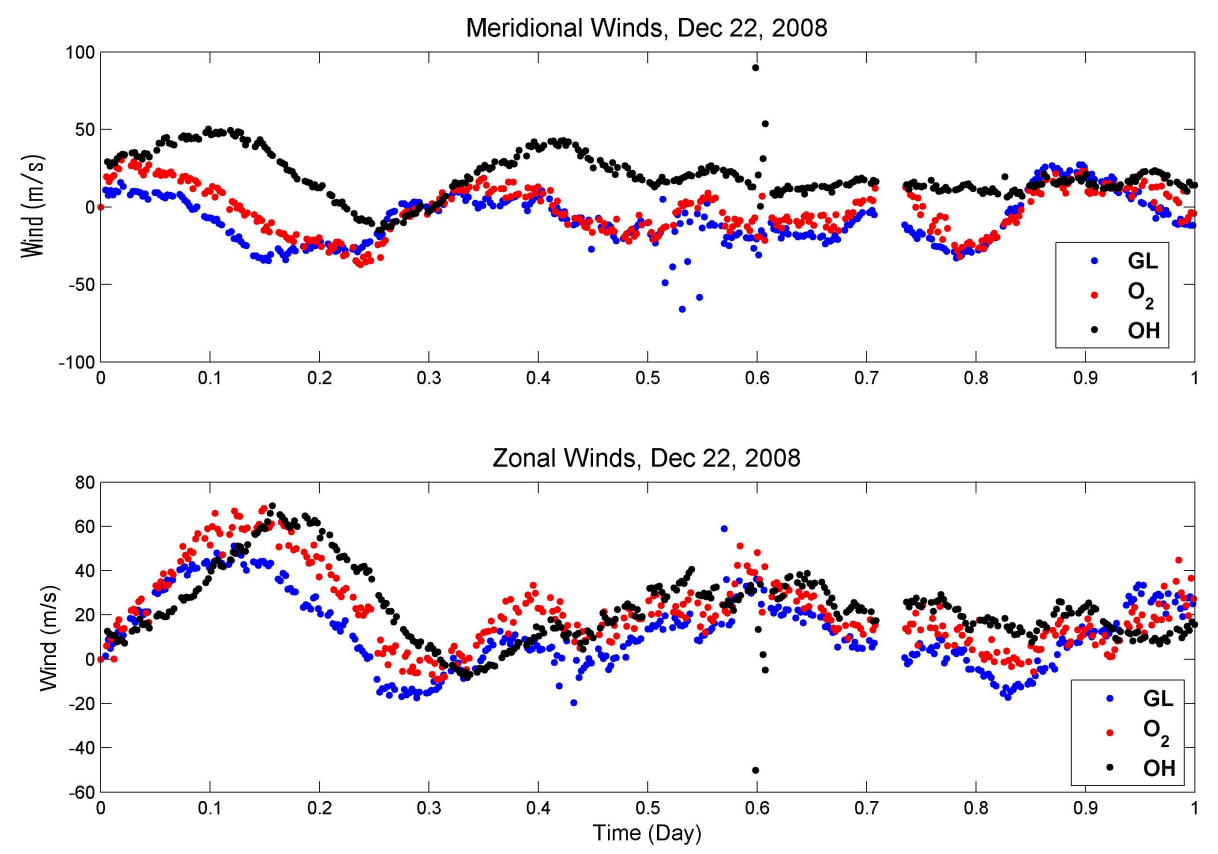

Fig. 11. Time series of the zonal and meridional winds for all three emissions on 22 December 2008. It is interesting to note that larger scale variations first show up in the green-line winds, followed by $\mathrm{O}_{2}$ and then $\mathrm{OH}$. This is expected for upward propagating tides for which the phase front propagates downward. Standard errors for each wind measurement are smaller than the dots in the figure for green line and $\mathrm{OH}$ and about the size of the dot for $\mathrm{O}_{2}$.

Frank et al. (2005) which compares lidar winds to meteor radar winds are quoted as being a few $\mathrm{m} \mathrm{s}^{-1}$ for (time/height) bins of $(60 \mathrm{~min} / 4 \mathrm{~km})$. For the lidar used in this study, vector winds were obtained with $\sim 1 \mathrm{~m} \mathrm{~s}^{-1}$ precision between 85 and $100 \mathrm{~km}$ with a $12 \mathrm{~min}$ cycle and vertical resolution of $\sim 1 \mathrm{~km}$ (Liu and Gardener, 2005).

As discussed by Franke et al. (2005) and Fujii et al. (2004), the geometries of the observing conditions associated with the various wind measuring instruments are different. They are sensitive to different temporal and spatial scales, so the measurement variances are affected differently by geophysical variability. ERWIN II views a brightness-weighted wind, so as noted in Sect. 3 it can be considered to provide winds for a volume of atmosphere of $\sim 5 \mathrm{~km}$ in the vertical and $5 \mathrm{~km}$ by $6 \mathrm{~km}$ in the horizontal In contrast, the lidar collects information from volumes with heights of $1 \mathrm{~km}$ and a diameter of $\sim 50 \mathrm{~m}$ and the meteor radar collects information per velocity measurement from a volume $4 \mathrm{~km}$ thick and $200 \mathrm{~km}$ diameter (Franke et al., 2005). The sampling area of the MU radar per velocity measurement is from a volume $1 \mathrm{~km}$ thick and $200 \mathrm{~km}$ in diameter. Based on the height range and vertical resolution of the best of these various instruments, the lidar achieves 15 velocity measurements at a $1 \mathrm{~m} \mathrm{~s}^{-1}$ precision in $12 \mathrm{~min}$, and the MU radar achieves 40 velocity measurements at a $3-5 \mathrm{~m} \mathrm{~s}^{-1}$ precision (recent upgrades to the MU radar may have enhanced its capabilities). With a vector wind measurement every $45 \mathrm{~s}$ at a precision of $\sim 1 \mathrm{~m} \mathrm{~s}^{-1}$, ERWIN II has similar capabilities to these instruments. For a cycle through the three emissions, winds at nominal heights of 7,93 and $97 \mathrm{~km}$ at the four cardinal directions and the vertical are obtained every three minutes at a precision equal to or better than these instruments and at a measurement cadence faster than either of these instruments. On the other hand, these instruments provide better spatial resolution than ERWIN II (especially in the vertical) since the observation volumes associated with each measurement are smaller.

Some indication of the capabilities of ERWIN II for scientific purposes is shown in Fig. 11. Here time series of the meridional and zonal winds observed by ERWIN II on 22 December 2008 for all three emissions are presented. As expected for larger scale upward propagating waves, the phase progression is downward with the green-line winds leading, followed by $\mathrm{O}_{2}$ and then $\mathrm{OH}$. Standard errors for each wind measurement are smaller than the dots in the figure for green line and $\mathrm{OH}$ and about the size of the dot for $\mathrm{O}_{2}$. The smaller scale variations are real and provide the opportunity to investigate the wind fields at these heights on smaller temporal scales than previously possible. Given that ERWIN II simultaneously provides observations of the relative brightness of the airglow emissions that it observes, the relationship between the winds and airglow can be explored in detail. Of particular interest is the investigation of gravity waves and the associated vertical velocities and airglow variations.

The PEARL facility, where ERWIN II is currently located, houses three other instruments which take measurements in 
the upper mesosphere and lower thermosphere. These include an All-Sky Imager (ASI) (observes $\mathrm{OH}, \mathrm{Na}, \mathrm{O}\left({ }^{1} \mathrm{~S}\right)$, $\mathrm{O}\left({ }^{1} \mathrm{D}\right)$ and $\left.\mathrm{N}_{2}^{+}\right)$, a spectral airglow temperature imager (SATI: $\mathrm{O}_{2}$, OH; Sargoytchev et al., 2004; Shepherd et al., 2010), and a meteor radar (Manson et al., 2009). The measurement cadences of the ASI and SATI are of the order of minutes, and the meteor radar provides an hourly vertical profile of horizontal wind in the mesopause region. Intercomparisons between simultaneous observations taken with these instruments open up many possibilities for new scientific studies. Initial comparisons between ERWIN II and the meteor radar show them to be in reasonable agreement (a comparison which deals with the complexities of the observational filters of each instrument will be published separately). The dynamical signatures of specific events (such as stratospheric warming), wave signatures and the relationships between temperature, wind and emission rate over a broad range of scales are topics which this research station is especially capable of investigating.

\section{Conclusions}

The construction of ERWIN II and the completion of new data analysis algorithms have resulted in a powerful new capability for investigating the dynamics of the mesopause region. The most important physical changes to the instrument include the addition of a quad mirror to the ERWIN optical train so that multiple viewing directions can be simultaneously observed and the inclusion of a CCD camera so that each of these directions can be simultaneously imaged. The new data analysis algorithm takes advantage of the imaging capabilities of the new instrument to provide a more precise and better monitored wind and volume emission rate observations.

In this paper the capabilities of this instrument have been thoroughly discussed. For the standard observation sequence, wind measurements have a precision and accuracy of $\sim 1 \mathrm{~m} \mathrm{~s}^{-1}$ and a 3 min observation cadence which incorporates observations in five viewing directions for each of three different emissions. This accuracy, precision and observing cadence was shown to be the best to date for optical instruments which use airglow to measure winds. Comparisons with superior radar and lidar systems indicate that ERWIN II wind observations are among the best in the world.

New science is anticipated with this instrument. On its own, vertical winds and relationships between the wind components and airglow volume emission rate can be investigated at temporal scales previously unachievable. Of particular interest in this respect is the investigation of the relationships between these variables in gravity waves and tides, and the investigation of the velocity spectra at these heights.

The installation of ERWIN II at PEARL, along with several other instruments which observe temperature, airglow and wind in the mesopause region, establishes a unique and potent capability both amongst Arctic observatories and worldwide. These instruments include a SATI, an All-Sky Imager and a meteor radar. Together they support the investigation of the spatial and temporal variability of the temperature, wind and airglow on temporal scales of minutes to months and spatial scales from kilometres to hundreds of kilometres.

Acknowledgements. The Canadian Network for the Detection of Atmospheric Change (CANDAC)/PEARL funding partners are as follows: the Arctic Research Infrastructure Fund, Atlantic Innovation Fund/Nova Scotia Research Innovation Trust, Canadian Foundation for Climate and Atmospheric Science, Canadian Foundation for Innovation, Canadian Space Agency, Environment Canada, Government of Canada International Polar Year, Natural Sciences and Engineering Research Council, Ontario Innovation Trust, Ontario Research Fund, Indian and Northern Affairs Canada, and the Polar Continental Shelf Program. Funds from the Canadian Foundation of Innovation were essential for the construction of the ERWIN II instrument. Research support through grants from the Natural Sciences and Engineering Council (NSERC), research funding from the Canadian Foundation for Climate and Atmospheric Science (CFCAS), and support from the CREATE program of NSERC as well as the University of New Brunswick and York University are acknowledged. Financial support for Stephen Brown was provided through the CRESS Space Instrumentation Laboratory (CSIL) at York University. Brian Solheim and Gordon Shepherd are also thanked for their support of the design and construction of ERWIN II.

Edited by: A. Stoffelen

\section{References}

Anderson, C., Conde, M., and McHarg, M. G.: Neutral thermospheric dynamics observed with two scanning Doppler imagers: 1. Monostatic and bistatic winds, J. Geophys. Res., 117, A03304, doi:10.1029/2011JA017041, 2012.

Aruliah, A. L., Griffin, E. M., Yiu, H.-C. I., McWhirter, I., and Charalambous, A.: SCANDI - an all-sky Doppler imager for studies of thermospheric spatial structure, Ann. Geophys., 28, 549-567, doi:10.5194/angeo-28-549-2010, 2010.

Bhattacharya, Y. and Gerrard, A. J.: Wintertime mesopause region vertical winds from Resolute Bay, J. Geophys. Res., 115, D00N07, doi:10.1029/2010JD014113, 2010.

Chu, Y. H., Su, C. L., Larsen, M. F., and Chao, C. K.: First measurements of neutral wind and turbulence in the mesosphere and lower thermosphere over Taiwan with a chemical release experiment, J. Geophys. Res., 112, A02301, doi:10.1029/2005JA011560, 2007.

Englert, C. R., Babcock, D. D., and Harlander, J. M.: Doppler Asymmetric Spatial Heterodyne Spectroscopy (DASH): concept and experimental demonstration, Appl. Optics, 46, 7297-7307, 2007. 
Englert, C. R., Harlander, J. M., Brown, C. M., Meriwether, J. W., Makela, J. J., Castelaz, M., Emmert, J. T., Drob, D. P., and Marr, K. D.: Coincident thermospheric wind measurements using ground-based Doppler Asymmetric Spatial Heterodyne (DASH) and Fabry-Perot Interferometer (FPI) instruments, J. Atmos. Sol.-Terr. Phy., 86, 92-98, 2012.

Fisher, G. M., Killeen, T. L., Wu, Q., Reeves, J. M., Hays, P. B., Gault, W. A., Brown, S., and Shepherd, G. G.: Polar cap mesosphere wind observations: Comparisons of simultaneous measurements with a Fabry-Perot interferometer and a field-widened Michelson interferometer, Appl. Optics, 39, 4284-4291, 2000.

Fisher, G. M., Niciejewski, R. J., Killeen, T. L., Gault, W. A., Shepherd, G. G., Brown, S., and Wu, Q.: Twelve-hour tides in the winter northern polar mesosphere and lower thermosphere, J. Geophys. Res., 107, 1211, doi:10.1029/2001JA000294, 2002.

Franke, S. J., Chu, X., Liu, A. Z., and Hocking, W. K.: Comparison of meteor radar and Na Doppler lidar measurements of winds in the mesopause region above Maui, Hawaii, J. Geophys. Res., 110, D09S02, doi:10.1029/2003JD004486, 2005.

Fujii, J., Nakamura, T., Tsuda, T., and Shiokawa, K.: Comparison of winds measured by MU radar and Fabry-Perot interferometer and effect of OI5577 airglow height variations, J. Atmos. Sol.Terr. Phy., 66, 573-583, 2004.

Gault, W. A., Brown, S., Moise, A., Liang, D., Sellar, G., Shepherd, G. G., and Wimperis, J.: ERWIN: An E region wind interferometer, Appl. Optics, 35, 2913-2922, doi:10.1364/AO.35.002913, 1996.

Hilliard, R. L. and Shepherd, G. G.: Wide-Angle Michelson Interferometer for measuring Doppler line widths, J. Opt. Soc. Am., 56, 362-369, 1966.

Kristoffersen, S. K.: The E-Region Wind Interferometer (ERWIN): Description of the Least Mean Squares Data Analysis Routine, Wind Results and Comparisons with the Meteor Radar, University of New Brunswick, Fredericton, NB, Canada, 2012.

Larsen, M. F.: Winds and shears in the mesosphere and lower thermosphere: Results from four decades of chemical release wind measurements, J. Geophys. Res., 107, doi:10.1029/2001JA000218, 2002.

Liu, A. Z. and Gardner, C. S.: Vertical heat and constituent transport in the mesopause region by dissipating gravity waves at Maui, Hawaii $\left(20.7^{\circ} \mathrm{N}\right)$, and Starfire Optical Range, New Mexico ( $\left.35^{\circ} \mathrm{N}\right)$, J. Geophys. Res., 110, D09S13, doi:10.1029/2004JD004965, 2005.

Makela, J. J., Meriwether, J. W., Huang, Y., and Sherwood, P. J.: Simulation and analysis of a multi-order imaging Fabry-Perot interferometer for the study of thermospheric winds and temperatures, Appl. Optics, 50, 4403-4416, 2011.

Manney, G. L., Schwartz, M. J., Krüger, K., Santee, M. L., Pawson, S., Lee, J. N., Daffer, W. H., Fuller, R. A., and Livesey, N. J.: Aura Microwave Limb Sounder observations of dynamics and transport during the record-breaking 2009 Arctic stratospheric major warming, Geophys. Res. Lett., 36, L12815, doi:10.1029/2009GL038586, 2009.
Manson, A. H., Meek, C. E., Chshyolkova, T., Xu, X., Aso, T., Drummond, J. R., Hall, C. M., Hocking, W. K., Jacobi, Ch., Tsutsumi, M., and Ward, W. E.: Arctic tidal characteristics at Eureka $\left(80^{\circ} \mathrm{N}, 86^{\circ} \mathrm{W}\right)$ and Svalbard $\left(78^{\circ} \mathrm{N}, 16^{\circ} \mathrm{E}\right)$ for $2006 / 07$ : seasonal and longitudinal variations, migrating and non-migrating tides, Ann. Geophys., 27, 1153-1173, doi:10.5194/angeo-271153-2009, 2009.

Meriwether, J. W., Makela, J. J., Huang, Y., Fisher, D. J., Buriti, R. A., Medeiros, A. F., and Takahashi, H.: Climatology of the nighttime equatorial thermospheric winds and temperatures over Brazil near solar minimum, J. Geophys. Res., 116, A04322, doi:10.1029/2011JA016477, 2011.

Press, W. H., Teukolsky, S. A., Vetterline, W. T., and Flannery, B. P.: Numerical Recipes The Art of Scientific Computing, 3rd Edn., Cambridge University Press, Cambridge, 2007.

Sargoytchev, S. I., Brown, S., Solheim, B. H., Cho, Y.-M., Shepherd, G. G., and López-González, M. J.: Spectral airglow temperature imager SATI: a ground-based instrument for the monitoring of mesosphere temperature, Appl. Optics, 43, 5712-5721, 2004.

She, C. Y., Sherman, J., Yuan, T., Williams, B. P., Arnold, K., Kawahara, T. D., Li, T., Xu, L. F., Vance, J. D., Acott, P., and Krueger, D. A.: The first 80-hour continuous lidar campaign for simultaneous observation of mesopause region temperature and wind, Geophys. Res. Lett., 30, 1319, doi:10.1029/2002GL016412, 2003.

Shepherd, G. G.: Spectral Imaging of the Atmosphere, Elsevier Academic Press, London, UK, 2002.

Shepherd, G. G., Thuillier, G., Gault, W. A., Solheim, B. H., Hersom, C., Alunni, J. M., Brun, J.-F., Charlot, P., Cogger, L. L., Desaulniers, D.-L., Evans, W. F. J., Gattingert, R. L., Girod, F., Harvie, D., Hum, R. H., Kendall, D. J. W., Llewellyn, E. J., Lowe, R. P., Ohrt, J., Pasternak, F., Peillet, O., Powell, I., Rochon, Y., Ward, W. E., Weins, R. H., and Wimperis, J.: WINDII, the wind imaging interferometer on the Upper Atmosphere Research Satellite, J. Geophys. Res., 98, 10725-10750, 1993.

Shepherd, M. G., Cho, Y.-M., Shepherd, G. G., Ward, W. E., and Drummond, J. R.: Mesospheric Temperature and Atomic Oxygen Response during the January 2009 Major Stratospheric Warming, J. Geophys. Res., 115, A07318, doi:10.1029/2009JA015172, 2010.

Shiokawa, K., Otsuka, Y., Oyama, S., Nozawa, S., Satoh, M., Katoh, Y., Hamaguchi, Y., and Yamamoto, Y.: Development of low-cost sky-scanning Fabry-Perot interferometers for airglow and auroral studies, Earth Planets Space, 64, 1033-1046, doi:10.5047/eps.2012.05.004, 2012.

Ward, W. E.: Design and Implementation of a Wide Angle Michelson Interferometer to Observe Thermospheric Winds, Ph.D. Thesis, York University, Toronto, Ont, Canada, 1988.

Zwick, H. H. and Shepherd, G. G.: Defocusing a Wind-Angle Michelson Interferometer, Appl. Optics, 10, 2569-2571, 1971. 